\section{Biomedical Reviews}

\section{INSTRUCTIONS TO AUTHORS}

\section{General Information}

Biomedical Reviews (BMR) is an official Journal of the Bulgarian Society for Cell Biology (BGSCB) published by the Medical University Press, Varna, Bulgaria. Biomedical Reviews publishes articles focused on updated knowledge in disease-oriented molecular cell biology. The following types of contributions are published:

(i) Review articles summarize state-of-the-science (SOS) on a given biomedical topic. Contributors to Reviews are, in general, invited by the Editors and the Editorial Board, but idea proposals are welcome. Potential authors are invited to submit a letter of interest to the Editor. Proposals should contain an outline of the contents, including an abstract, a list of 20 relevant articles including from the proposer's own research, and a brief statement on why now is a good time to review the topic in question. Reviews will not be accepted for editorial processing unless pre-approved for submission.

(ii) Dance Round articles are short, position papers that are intended to focus observations that seem to point the field in a new direction, to give the author's personal views on a controversial topic, or to direct soundly based criticism at some widely held dogma in biomedicine.

(iii) Research articles, and (iv) Topic issues aimed at clustering contributions to a biomedical cutting edge within one issue. Guest Editors of such issues are, in general, invited by the Editors and the Editorial Board, but idea proposals are welcome. Multiple-part papers are discouraged. Manuscripts submitted under multiple authorship are reviewed with the understanding that all listed authors concur in the submission and that the final manuscript has been approved by all authors. If accepted, the article shall not be published elsewhere in the same form, in either the same or another language, without the written consent of the Editors and Publisher.

\section{Organization of the Manuscript}

Text of manuscripts must include an abstract, an introduction, followed by the body of manuscript, a conclusion, acknow- ledgments, a list of references, and, if available, figure legends, and tables. Pages should be double-spaced, Times New Roman should be used throughout, sized at $12 \mathrm{pt}$. The text file should be submitted in either Word or PDF format to the Editor-in-Chief at chaldakov@yahoo.com and/or to some of the Editors at his e-mail indicated on the inside front cover.

\section{Title Page}

Please organize a title page as the first page of the text file to include the following:

- Title

- Abbreviated title

- Authors and their affiliations.

- Corresponding author with complete address, including telephone and fax number, and an e-mail address

- Number of figures, tables and pages

- Up to 6 key words that do not appear in the title

\begin{abstract}
The abstract (typically about, although not strictly restricted to, 250 words) should provide a concise summary of the data to be reviewed and major conclusions of the study. It should be written in complete sentences, without explicit subheadings. Citing references should be avoided.
\end{abstract}

\section{Introduction}

The introduction should briefly indicate the background of the topic, and explain the objectives of the paper.

Captions should be used within the body of the manuscript to outline important points.

\section{Conclusion}

This section should be as concise as possible and should summarize the data discussed in the paper, and possibly, should contain a statement of their significance and future biomedical implications.

\section{References}

Only published and "in press" references should appear in the reference list. The latest information on "in press" references should be provided. Any "in press" references that are relevant for reviewers to see in order to make a well-informed evaluation should be included as a separate document text file along with the submitted manuscript. "Submitted" references as well as personal communications should be cited only in text. Authors are responsible for all personal communications and must obtain written approval from persons cited before submitting the paper to the Journal. Proof of such approval may be requested by the Journal.

References should be each numbered, ordered sequentially as they appear in the text, and cited in parentheses: "text (1)". In 
case of with multiple references, these should be cited starting from the smallest number: "text (1-3)". In the list of references, papers should be listed numerically. The name (surname first) of the author(s) should be followed the by the full title of the paper as it appeared in the original, the source of the reference, together with the year, volume number, and the first and last pages. If the author list for a paper exceeds 6, et al (in oblique font) should be added after the sixth author. References to web-only journals should also provide URL in full or DOI if known. Book titles are in oblique font with all main words' first letter being capitalized. The following illustrates the format to be used:

\section{- Journal article}

Iwamoto Y, Koide H, Ogita K, Nishizuka Y. The protein kinase $\mathrm{C}$ family for the regulation of cellular functions. Biomed Rev 1992; 1: 1-6.

\section{- Book}

Author A. Book Title. Publisher name, 2000.

\section{- Chapter in a book}

Author A. Chapter title. In: Author A, editor(s). Book Title. Publisher name, 2000; 1-10.

Abbreviations of journal titles should follow those listed in the Index Medicus. Responsibility for the correctness of the references lies with the author(s). After manuscript revisions, authors should double check that all in-text citations are in the reference list and that all references on the reference list have at least one corresponding in-text citation.

\section{Illustrations}

All figures must be cited in the text and numbered consecutively (Fig. 1, Fig. 2, etc.). Each figure should be submitted as a separate file. For vector graphics, EPS (Encapsulated PostScript) files are the preferred format. TIFF (Tagged Image File Format) is the recommended file format for bitmap, greyscale and colour images. When supplying TIFF files please ensure that files are supplied at the correct resolution:

- line artwork = minimum of $1000 \mathrm{ppi}$

- halftone artwork = minimum of $300 \mathrm{ppi}$
- combination artwork (line $/$ tone $)=$ minimum of $500 \mathrm{ppi}$ Authors should be aware that using color figures will incur an additional charge for color in their reprints.

\section{Preparation of Tables}

Each table should be double-spaced with an explanatory title and will appear at the end of the text of the manuscript. All tables must be cited in the text (e.g. "Table 1").

\section{Figure Legends}

Each figure should be accompanied by a title and an explanatory legend. The title should be part of the legend and not lettered onto the figure itself. Legends should be concise.

\section{Abbreviations}

Use abbreviations if a term appears three or more times. Spell out all abbreviations at first occurrence, and then introduce them by placing the abbreviation in parentheses. The metric system should be used for all volumes, lengths, weights, etc. Temperatures should be expressed in degrees Celsius (centigrade). Units should conform to the International System of Units (SI).

\section{PROOFS, OFFPRINTS, MISCELLANEOUS}

\section{Proofs}

Proofs will be sent by E-mail, as PDFs. No major changes in, or additions to, the edited manuscript will be allowed at this stage. Proofreading is solely the Authors' responsibility. Corrections to the proofs must be returned by E-mail or Fax within 72 hours after receipt. If the Publisher receives no response from the Author(s) after 10 days, it will be assumed that there are no errors to correct and the article will be published.

\section{Page charges}

There are no page charges.

Abstracted in CAB Abstracts/Global Health databases, Chemical Abstracts, Excerpta Medica database (EMBASE), Compendex, EMBiology, Elsevier BIOBASE, Index Cope- rnicus International, Index Scholar, and Scopus.

Biomedical Reviews is published annually by Medical University Press, Varna, Bulgaria on behalf of the Bulgarian Society for Cell Biology, a professional society serving the cell biology community

www.bgscb.org/BMR.htm

http://press.mu-varna.bg/ojs/ 